ACTA MYCOLOGICA

Vol. 42 (1): 21-34

2007
Dedicated to Professor Alina Skirgietto

on the occasion of her ninety-fifth birthday

\title{
Macromycetes in the Pirin Mts (SW Bulgaria)
}

\author{
CVETOMIR M. DENCHEV, VIOLETA I. FAKIROVA, MELANIA M. GYOSHEVA \\ and ROUMYANA D. PETROVA
}

Bulgarian Mycological Society

23 Acad. G. Bonchev St., BU-1113 Sofia, cmdenchev@yahoo.co.uk

Denchev C. M.,Fakirova V. I., Gyosheva M. M., Petrova R. D.: Macromycetes in the Pirin Mts (SW Bulgaria). Acta Mycol. 42 (1): 21-34, 2007.

This paper attempts to compile available and unpublished data on the macromycetes of the Pirin Mts. The total number of currently known species is 350, including 34 ascomycetes and 316 basidiomycetes. Among them, 60 species are reported for the first time for the Pirin Mts, including Inocybe pisciodora which is a new record for Bulgaria. The species composition, ecological-trophic structure, and economically important edible fungi are briefly discussed. A list of 25 macroscopic fungi of conservation significance for the mycota of this mountain, evaluated with the recent IUCN criteria, is given, including 4 Critically Endangered, 9 Endangered, 7 Vulnerable, and 5 Near Threatened species.

Key words: Bulgaria, fungal diversity, macromycetes, Pirin Mts, Pirin National Park

\section{INTRODUCTION}

Pirin Mts is situated in the south-western part of Bulgaria. In this article, the Pirin Mts refers to the floristic region of Pirin, according to Jordanov (1966) (Fig. 1). The Pirin National Park, on the area of 40332 ha (57\% of which covered by forest plant communities), is located in the territory of that mountain. There are two reserves in the Park, Bayuvi Doupki-Dzhindzhiritsa Reserve (2873 ha) and Yulen Reserve (3156 ha). Regarding the flora, the Pirin Mts is one of the most interesting Bulgarian and Balkan mountains. The main tree species in the forest plant communities are Fagus sylvatica, Pinus nigra, P. sylvestris, P. peuce, P. heldreichii, Picea abies, and Pinus mugo.

Unfortunately, the inventory of Pirin Mts mycota is still far behind comparing to the inventory of the flora and fauna. There is no published list of macromycetes as for the whole mountain as well as for the Pirin National Park in particular. The available information is scanty and referes mainly to short communications on establishment of new or rare species for Bulgaria. Data on macromycetes of the Pirin Mts are published by Pilát (1926); Burzakov (1928, 1932); Kre is el (1959); Hin kova (1961, 


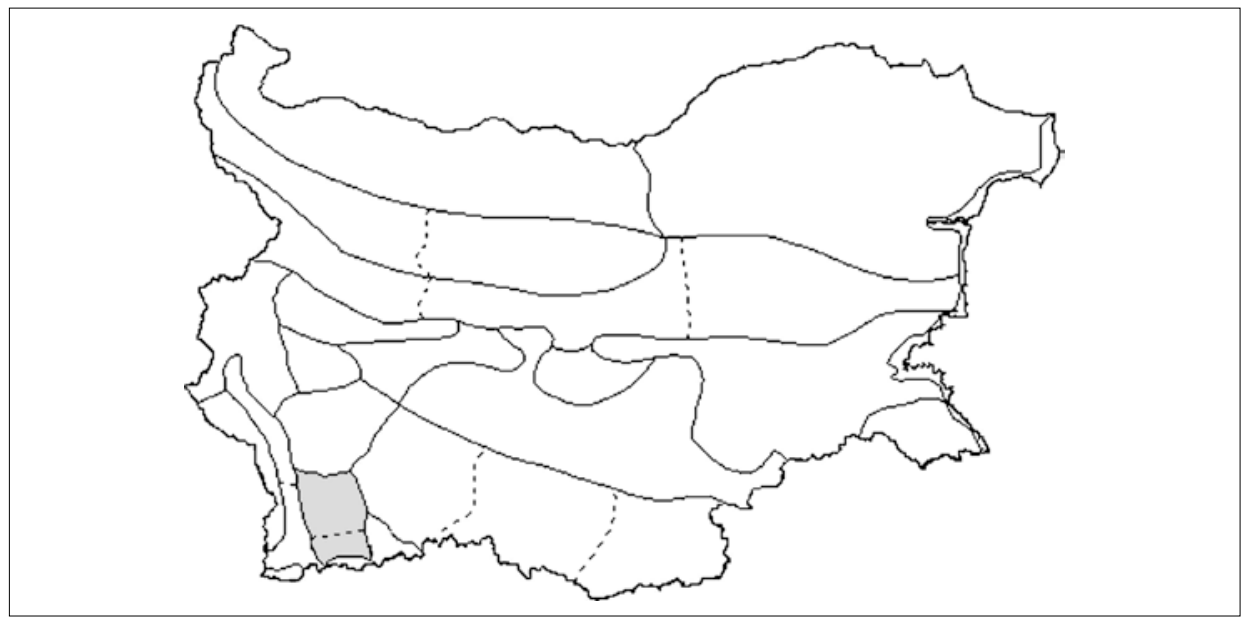

Fig. 1. Floristic regions in Bulgaria (by J or da nov 1966).

The Pirin Mts (northern and southern parts) are highlighted.

1965); Dörfelt (1977); Chaluk ov (1978, 1982, 1984, 1985, 1987); S to ich ev (1981, 1987a, b, 1990, 1995); S toichev and Naidenov (1984); Vanev and Reid (1986); Dimcheva and Stoichev (1987); Dörfelt and Müsch (1987); Stoichev and Dimcheva (1987, 1988); Kuthan and Kotlaba (1988); Drumeva-Dimcheva and Gyos heva-Bogoeva (1993); Dimitrova (1994, 1997, 1998, 1999, 2000); La Porta et al. (1998); Gyosheva (2000); Gyosheva et al. (2000); Assyov (2004); Assyov and Denchev (2004); Lacheva and Stoichev (2004). Among them, only Dim cheva and Stoichev (1987), basing on the available information for the species diversity, attempted to analyse the trophic groups of macromycetes in the mountain. Drumeva-Dimcheva and Gyosheva-Bogoeva (1993) reported data on the macromycetes in Bayuvi Doupki-Dzhindzhiritsa Reserve. Orderly mycological studies on the mountain and Pirin National Park, in particular, have not been carried out yet. There are no complete collections of specimens from the Pirin reserves, as well as from some areas such as massifs, river basins, etc. All available materials have been gathered on short mycological trips, mainly following the traditional tourist routes in the valleys of Bunderitsa River and Demyanitsa River and in the area of Yavorov Chalet. No orderly ecological studies have been carried out for a period of several years.

In 2001-2003 the Management Plan of the Pirin National Park was prepared. Its chapter devoted to the fungal diversity was worked out by a group of the Bulgarian Mycological Society, including the first two authors of the present article. For this purpose, during 2001-2002 short field investigations were conducted and all the available information about the mountain was analyzed.

In the present article, for the first time, all published records on macromycetes from the Pirin Mts and, in particular, from the Pirin National Park, as well as data from the field investigations in 2001-2002 and other unpublished data, obtained from the public mycological collections in Sofia (SOMF) and Plovdiv (SOA), are summarized and briefly discussed. The taxonomic and chorological information about Pirin 
Mts is still incomplete for a serious analysis of the taxonomic structure as well as for the objective estimation of the status of species threat and the anthropogenic influence on economically important edible mushrooms

\section{MATERIALS AND METHODS}

In Table 1, lists of macroscopic fungi of the Pirin Mts and separately, of the Pirin National Park, are given. The listed taxa within ascomycetes and basidiomycetes are given in alphabetical order. The authors' names are abbreviated according to Kirk et al. (2004). The information in the presented lists includes available data from literature sources, unpublished data of the authors, and data from the Mycological Collection of the Institute of Botany, Sofia (SOMF), and Mycological Collection of the Agricultural University, Plovdiv (SOA). The treatment of different genera and species follows a lot of recent monographs and particular articles on European fungi. Taxonomic judgements on unexamined specimens were avoided. A brief list of published synonyms, is listed separately, with references to the names used in the main list in Table 1.

The conservation status of the species is based on the contemporary and official Red List of Fungi in Bulgaria (Gyosheva et al. 2006), where the current IUCN Red Data Book categories (IUCN 2001, 2003a, b) are put into practice.

Table 1

Checklist of macromycetes in the Pirin Mts

\begin{tabular}{|c|c|c|c|c|c|}
\hline & Taxa & Park** & ETG**** & $\mathrm{C}^{* * * * *}$ & Sources***** \\
\hline & ASCOMYCOTA & & & & \\
\hline 1 & Aleuria aurantia (Pers.) Fuckel* & + & $\mathrm{Hu}$ & & SOMF \\
\hline 2 & Brunnipila clandestina (Bull. : Fr.) Baral & + & Fd & & 41 \\
\hline 3 & Caloscypha fulgens (Pers. : Fr.) Boud.* & + & $\mathrm{Hu}, \mathrm{LeS}$ & & SOMF \\
\hline 4 & Ciboria batschiana (Zopf) N.F. Buchw. & + & $\mathrm{Fd}$ & & 12, SOMF \\
\hline 5 & Crocicreas cyathoideum (Bull.) S.E. Carp. & + & $\mathrm{Fd}$ & & $13, \mathrm{SOMF}$ \\
\hline 6 & Cudoniella clavus (Alb. \& Schwein.) Dennis & + & $\mathrm{Fd}$ & & 41 \\
\hline 7 & Dasyscyphus mollissimus (Lasch) Dennis & + & $\mathrm{Fd}$ & & 41, SOMF \\
\hline 8 & D. oblongosporus G.G. Hahn \& Ayers & + & LeS & & 11,14, SOMF \\
\hline 9 & Dermea piceina J.W. Groves & + & LeS & & 12,14, SOMF \\
\hline 10 & Discina ancilis (Pers.) Sacc. & + & LeS & VU & 10,21 \\
\hline 11 & Duplicaria empetri (Pers. : Fr.) Fuckel & + & Fd & & 22 \\
\hline 12 & Elaphomyces granulatus Sacc. \& Bizz. & & $\mathrm{Mr}$ & CR & 19,21, SOMF \\
\hline 13 & Gyromitra esculenta (Pers. : Fr.) Fr.* & + & $\mathrm{Hu}$ & & unpubl. \\
\hline 14 & G. infula (Schaeff. : Fr.) Quél. & & LeS & & 23 \\
\hline 15 & Helvella acetabulum (L.) Quél.* & + & $\mathrm{Hu}$ & & SOMF \\
\hline 16 & H. lacunosa Afzel. : Fr.** & & $\mathrm{Hu}$ & & SOMF \\
\hline 17 & Herpotrichia juniperi (Duby) Petr. & + & Ad & & 41 \\
\hline 18 & Hymenoscyphus imberbis (Bull.) Dennis & & LeS & & 43 \\
\hline 19 & Hysterium acuminatum Fr. : Fr. & + & LeS & & 41 \\
\hline 20 & Lachnum bicolor (Bull. : Fr.) P. Karst. & + & Fd & & 15,41 \\
\hline 21 & L. brevipilosum Baral & + & LeS & & 15 \\
\hline 22 & L. virgineum (Batsch : Fr.) P. Karst. & + & $\mathrm{S}$ & & 15,41 \\
\hline 23 & $\begin{array}{l}\text { Lasiobelonium nidulum (J.C. Schmidt \& } \\
\text { Kunze) Spooner }\end{array}$ & & LeS & & 41 \\
\hline 24 & Morchella elata Fr. : Fr. & + & $\mathrm{Hu}$ & NT & 10,21 \\
\hline 25 & M. esculenta (L. : Fr.) Pers. & + & $\mathrm{Hu}$ & & 10,31 \\
\hline 26 & Otidea onotica (Pers. : Fr.) Fuckel & + & $\mathrm{Hu}$ & VU & 21,23 \\
\hline 27 & Peziza echinospora P. Karst. & + & Carb & & 10,41 \\
\hline
\end{tabular}


Tab. 1 cont.

\begin{tabular}{|c|c|c|c|c|c|}
\hline 28 & P. moseri Aviz.-Hersh. \& Nemlich & + & Carb & & 10,41 \\
\hline 29 & P. praetervisa Bres. & + & Carb & & 41 \\
\hline 30 & Pseudoplectania nigrella (Pers. : Fr.) Fuckel & + & $\mathrm{Br}$ & & 10 \\
\hline 31 & Sarcoscypha coccinea (Jaqc. : Fr.) Sacc.* & & LeS & & SOMF \\
\hline 32 & Spathularia flavida Pers. : Fr. & & St & NT & 20,21 \\
\hline 33 & Urnula craterium (Schwein. : Fr.) Fr. & + & St, LeS & $\mathrm{VU}$ & 10,21 \\
\hline \multirow[t]{2}{*}{34} & Xylaria polymorpha (Pers. : Fr.) Grev. & & LeS & & 43 \\
\hline & BASIDIOMYCOTA & & & & \\
\hline 35 & $\begin{array}{l}\text { Abortiporus biennis (Bull. : Fr.) Singer (= } \\
\text { Daedalea biennis) }\end{array}$ & & LeS & & 23,38, SOMF \\
\hline 36 & Agaricus arvensis Schaeff. : Fr. & + & $\mathrm{Hu}$ & & 3,10 \\
\hline 37 & A. augustus Fr.* & & & & SOMF \\
\hline 38 & A. campestris L. : Fr.* & + & $\mathrm{Hu}$ & & unpubl. \\
\hline 39 & A. silvaticus Schaeff. & + & $\mathrm{Hu}$ & & 10, SOMF \\
\hline 40 & A. subfloccosus (J.E. Lange) Pilát & + & & & 32 \\
\hline 41 & Albatrellus confluens (Fr.) Kotl. \& Pouzar & + & $\mathrm{Hu}$ & & 23 \\
\hline 42 & A. cristatus (Schaeff. : Fr.) Kotl. \& Pouzar & + & $\mathrm{Hu}$ & & 17,20, SOMF \\
\hline 43 & A. ovinus (Schaeff. : Fr.) Kotl. \& Pouzar & + & $\mathrm{Hu}$ & & 37, SOA \\
\hline 44 & Amanita battarrae (Boud.) Bon & + & $\mathrm{Mr}$ & & 17,20 \\
\hline 45 & A. citrina (Schaeff.) Pers. & + & $\mathrm{Mr}$ & & 17 \\
\hline 46 & A. gemmata (Fr.) Bertill. & + & $\mathrm{Mr}$ & & 17 \\
\hline 47 & $\begin{array}{l}\text { A. lividopallescens (Secr. ex Boud.) Kühner } \\
\& \text { Romagn. }\end{array}$ & + & $\mathrm{Mr}$ & & $10, \mathrm{SOMF}$ \\
\hline 48 & A. muscaria (L. : Fr.) Hook.* & + & $\mathrm{Mr}$ & & SOMF \\
\hline 49 & A. vaginata (Bull. : Fr.) Vittad. & + & $\mathrm{Mr}$ & & 18, SOMF \\
\hline 50 & $\begin{array}{l}\text { Amyloporia xantha }(\mathrm{Fr} .) \text { Bondartsev \& } \\
\text { Singer ex Bondartsev }\end{array}$ & + & LeS & & 10, SOA \\
\hline 51 & Antrodia serialis (Fr. : Fr.) Donk & + & LeS & & 10,38 \\
\hline 52 & A. xantha (Fr. : Fr.) Ryvarden & & LeS & & 38 \\
\hline 53 & Armillaria mellea (Vahl : Fr.) P. Kumm. & + & LeS, LeP & & 10 \\
\hline 54 & Asterophora parasitica (Bull. : Fr.) Singer & + & $\mathrm{M}$ & & 3 \\
\hline 55 & Astraeus hygrometricus (Pers. : Fr.) Morgan & + & $\mathrm{Hu}$ & & 17, SOMF \\
\hline 56 & Auriscalpium vulgare Gray & + & $\mathrm{S}$ & EN & 10,21, SOMF \\
\hline 57 & Bjerkandera adusta (Willd. : Fr.) P. Karst. & + & LeS & & $\begin{array}{l}\text { 10, 17, 37, } \\
\text { SOA }\end{array}$ \\
\hline 58 & B. fumosa (Pers. : Fr.) P. Karst & & LeS & & 37,38, SOA \\
\hline 59 & Bolbitius vitellinus (Pers. : Fr.) Fr.* & & $\mathrm{Hu}$ & & SOMF \\
\hline 60 & Boletus calopus Fr. & + & $\mathrm{Mr}$ & & $2,17, \mathrm{SOMF}$ \\
\hline 61 & B. chrysenteron Bull. & + & $\mathrm{Mr}$ & & $2,18, \mathrm{SOMF}$ \\
\hline 62 & B. edulis Bull. : Fr. & + & $\mathrm{Mr}$ & & 2,18, SOMF \\
\hline 63 & B. erythropus (Fr. : Fr.) Krombh. & + & $\mathrm{Mr}$ & & $2,17, \mathrm{SOMF}$ \\
\hline 64 & B. ferrugineus Schaeff. & + & $\mathrm{Mr}$ & & 18 \\
\hline 65 & B. luridus Schaeff. : Fr. & + & $\mathrm{Mr}$ & & $2,17, \mathrm{SOMF}$ \\
\hline 66 & B. pinophilus Pilát \& Dermek & + & $\mathrm{Mr}$ & & 2,18 \\
\hline 67 & B. queletii Schulzer s. lat. & + & $\mathrm{Mr}$ & & 2,23 \\
\hline 68 & B. subtomentosus Pers. : Fr. & + & $\mathrm{Mr}$ & & 2,17 \\
\hline 69 & Bovista nigrescens Pers. : Pers. & + & $\mathrm{Hu}$ & & 7, SOMF \\
\hline 70 & B. plumbea Pers. : Pers. & + & $\mathrm{Hu}$ & & 10, SOMF \\
\hline 71 & Calocera viscosa (Pers. : Fr.) Fr. & + & LeS & & 17 \\
\hline 72 & Cantharellus cibarius Fr. : Fr. & + & $\mathrm{Mr}$ & & $18, \mathrm{SOMF}$ \\
\hline 73 & C. tubaeformis (Bull. : Fr.) Fr. & & $\mathrm{Mr}$ & & 23 \\
\hline 74 & Cerrena unicolor (Bull. : Fr.) Murrill & & LeS & & 38 \\
\hline 75 & Chalciporus piperatus (Bull. : Fr.) Bataille & + & $\mathrm{Mr}$ & & $2,18, \mathrm{SOMF}$ \\
\hline 76 & Chlorophyllum rhacodes (Vittad.) Vellinga & + & $\mathrm{Hu}$ & & $\begin{array}{l}\text { 3, 10, 17, 34, } \\
\text { SOMF }\end{array}$ \\
\hline 77 & $\begin{array}{l}\text { Chroogomphus helveticus (Singer) M.M. } \\
\text { Moser }\end{array}$ & + & $\mathrm{Mr}$ & & $2,16,17$ \\
\hline 78 & Ch. rutilus (Schaeff. : Fr.) O.K. Mill. & + & $\mathrm{Mr}$ & & $2,17,18$ \\
\hline 79 & $\begin{array}{l}\text { Chrysomphalina chrysophylla (Fr.) } \\
\text { Clémençon }\end{array}$ & + & LeS & & 43 \\
\hline
\end{tabular}


Tab. 1 cont.

\begin{tabular}{|c|c|c|c|c|c|}
\hline 80 & Claudopus variabilis (Pers. : Fr.) Fr.* & + & LeS & & SOMF \\
\hline 81 & Clavariadelphus ligula (Schaeff. : Fr.) Donk & & $\mathrm{Mr}$ & VU & $\begin{array}{l}\text { 20, 21, 23, } \\
\text { SOMF }\end{array}$ \\
\hline 82 & Clavulina rugosa (Bull.) J. Schröt. & & $\mathrm{Hu}$ & & 23, SOMF \\
\hline 83 & $\begin{array}{l}\text { Climacocystis borealis (Fr. : Fr.) Kotl. \& } \\
\text { Pouzar }\end{array}$ & + & $\mathrm{LeS}$ & & 17 \\
\hline 84 & Clitocybe clavipes (Pers. : Fr.) P. Kumm. & + & $\mathrm{St}, \mathrm{Hu}$ & & 10,18, SOMF \\
\hline 85 & C. gibba (Pers. : Fr.) P. Kumm. & + & $\mathrm{Hu}$ & & 3,10, SOMF \\
\hline 86 & C. nebularis (Batsch : Fr.) Quél. & + & $\mathrm{Hu}$ & & 10,23 \\
\hline 87 & C. odora (Bull.) Fr. & + & St & & 17, SOMF \\
\hline 88 & C. radicellata Gillet & + & St & & 10, SOMF \\
\hline 89 & C. suaveolens (Schumach.) Fr.* & + & St & & SOMF \\
\hline 90 & C. vermicularis (Fr.) Gillet & + & $\mathrm{Ad}, \mathrm{St}$ & EN & $10,31, \mathrm{SOMF}$ \\
\hline 91 & Clitopilus prunulus (Scop. : Fr.) Fr.* & & $\mathrm{Hu}$ & & SOMF \\
\hline 92 & Coltricia perennis (L. : Fr.) Murrill & + & LeS & & 3,17 \\
\hline 93 & Conocybe blattaria (Fr. : Fr.) Kühner & + & $\mathrm{Hu}$ & & 41, SOMF \\
\hline 94 & C. juniana (Velen.) Hauskn. \& Svrček & + & $\mathrm{Hu}$ & & 43 \\
\hline 95 & C. pubescens (Gillet) Kühner & + & $\mathrm{C}$ & & 10 \\
\hline 96 & Coprinus atramentarius (Bull. : Fr.) Fr.* & + & $\mathrm{Hu}, \mathrm{LeS}$ & & SOMF \\
\hline 97 & C. laanii Kits van Wav.* & + & LeS & & unpubl. \\
\hline 98 & C. lagopus (Fr. : Fr.) Fr. & & $\mathrm{Hu}$ & & 43 \\
\hline 99 & C. radians (Desm. : Fr.) Fr.* & + & LeS & & unpubl. \\
\hline 100 & C. radiatus (Bolton) Gray & + & $\mathrm{C}$ & & 43 \\
\hline 101 & C. xanthothrix Romagn.* & + & LeS & & unpubl. \\
\hline 102 & Coriolopsis gallica (Fr.) Ryvarden & & LeS & & 37, SOA \\
\hline 103 & Cortinarius croceus (Schaeff.) Gray & + & $\mathrm{Mr}$ & & 10,18 \\
\hline 104 & C. elegantior (Fr.) Fr. & + & $\mathrm{Mr}$ & & 43 \\
\hline 105 & C. glaucopus (Schaeff. : Fr.) Fr. & + & $\mathrm{Mr}$ & & 41 \\
\hline 106 & C. largus Fr. & + & $\mathrm{Mr}$ & & 41, SOMF \\
\hline 107 & C. lustratus Fr.* & + & $\mathrm{Mr}$ & & SOMF \\
\hline 108 & C. sanguineus (Wulfen) Fr. & & $\mathrm{Mr}$ & & 10,41 \\
\hline 109 & C. traganus (Fr.) Fr. & + & $\mathrm{Mr}$ & & 17,41 \\
\hline 110 & C. venetus (Fr.) Fr.* & + & $\mathrm{Mr}$ & & SOMF \\
\hline 111 & Cyathus striatus (Huds. : Pers.) Hoffm. & + & LeS & & 17 \\
\hline 112 & $\begin{array}{l}\text { Cystoderma amianthinum (Scop. : Fr.) } \\
\text { Fayod }\end{array}$ & + & $\mathrm{Br}$ & & 10, SOMF \\
\hline 113 & C. carcharias (Pers.) Fayod & + & St & & 10,23, SOMF \\
\hline 114 & C. cinnabarinum (Alb. \& Schwein.) Fayod & & $\mathrm{St}, \mathrm{Hu}$ & & 23, SOMF \\
\hline 115 & C. granulosum (Batsch : Fr.) Fayod & + & St & & 10, SOMF \\
\hline 116 & Dacrymyces chrysocomus (Bull. : Fr.) Tul. & + & LeS & & 4 \\
\hline 117 & $\begin{array}{l}\text { Daedaleopsis confragosa (Bolton : Fr.) J. } \\
\text { Schröt. }\end{array}$ & & $\mathrm{LeS}$ & & 37, SOA \\
\hline 118 & Datronia mollis (Sommerf. : Fr.) Donk & & LeS & & 37, SOA \\
\hline 119 & Endoptychum agaricoides Czern. & + & $\mathrm{Hu}$ & EN & 10,21 \\
\hline 120 & $\begin{array}{l}\text { Entoloma hirtipes (Schumach. : Fr.) M.M. } \\
\text { Moser }\end{array}$ & + & $\mathrm{Mr}$ & & 41 \\
\hline 121 & E. mammosum (L.) Hesler & + & $\mathrm{Hu}$ & & 10, SOMF \\
\hline 122 & Fomitopsis pinicola (Sw. : Fr.) P. Karst. & + & LeP & & 17,28 \\
\hline 123 & Funalia trogii (Berk.) Bondartsev \& Singer & & LeS & & 38 \\
\hline 124 & Galerina marginata (Batsch : Fr.) Kühner & + & LeS & & 17 \\
\hline 125 & G. vittiformis (Fr.) Earle & + & LeS & & 28 \\
\hline 126 & Ganoderma applanatum (Pers.) Pat. & + & LeP, LeS & & 10,17 \\
\hline 127 & G. carnosum Pat. & + & LeS & & 17 \\
\hline 128 & G. lucidum (Curtis : Fr.) P. Karst. & + & LeS & & 42 \\
\hline 129 & G. resinaceum Boud. & & LeP, LeS & & 42 \\
\hline 130 & Geastrum fimbriatum Fr. & + & $\mathrm{Hu}$ & & $17,28,31$ \\
\hline 131 & G. melanocephalum (Czern.) V.J. Stanĕk & + & $\mathrm{Hu}$ & VU & $20,21,23$ \\
\hline 132 & G. pectinatum Pers. : Pers.* & + & $\mathrm{Hu}$ & & SOMF \\
\hline 133 & G. rufescens Pers. : Pers. & + & $\mathrm{Hu}$ & & 31 \\
\hline 134 & G. schmidelii Vittad. & & $\mathrm{Hu}$ & & 23 \\
\hline
\end{tabular}


Tab. 1 cont.

\begin{tabular}{|c|c|c|c|c|c|}
\hline 135 & $\begin{array}{l}\text { Gloeophyllum abietinum (Bull. : Fr.) P. } \\
\text { Karst. }\end{array}$ & + & LeS & & $10,17,34$ \\
\hline 136 & G. odoratum (Wulfen : Fr.) Imazeki & + & LeS & & 10 \\
\hline 137 & G. sepiarium (Wulfen : Fr.) P. Karst. & + & LeS & & $3,10,17$ \\
\hline 138 & Gomphidius glutinosus (Schaeff. : Fr.) Fr. & + & $\mathrm{Mr}$ & & 2,3, SOMF \\
\hline 139 & Guepinia helvelloides (DC. : Fr.) Fr. & + & LeS & EN & $20,21,22$ \\
\hline 140 & $\begin{array}{l}\text { Gymnopus peronatus (Bolton : Fr.) } \\
\text { Antonín, Halling \& Noordel. }\end{array}$ & & St & & 10, SOMF \\
\hline 141 & Gyrodon lividus (Bull. : Fr.) Sacc. & & $\mathrm{Mr}$ & $\mathrm{CR}$ & $2,20,21,23$ \\
\hline 142 & Gyroporus castaneus (Bull. : Fr.) Quél. & + & $\mathrm{Mr}$ & & 2, SOMF \\
\hline 143 & G. cyanescens (Bull. : Fr.) Quél. & + & $\mathrm{Mr}$ & & $2,17,18$ \\
\hline 144 & $\begin{array}{l}\text { Handkea excipuliformis (Scop. : Pers.) } \\
\text { Pers. }\end{array}$ & + & $\mathrm{Hu}$ & & SOMF \\
\hline 145 & H. utriformis (Bull. : Pers.) Pers.* & + & $\mathrm{Hu}$ & & SOMF \\
\hline 146 & Hebeloma circinans (Quél.) Sacc.* & + & $\mathrm{Mr}$ & & SOMF \\
\hline 147 & H. edurum Métrod ex Bon* & + & $\mathrm{Mr}$ & & SOMF \\
\hline 148 & Hemimycena gracilis (Quél.) Singer & + & $\mathrm{Ad}$ & & 43 \\
\hline 149 & Heterobasidion annosum (Fr. : Fr.) Bref. & + & LeP & & $10,17,33$ \\
\hline 150 & Hydnellum ferrugineum (Fr. : Fr.) P. Karst.* & + & $\mathrm{Mr}$ & & SOMF \\
\hline 151 & H. suaveolens (Scop. : Fr.) P. Karst. & + & $\mathrm{Mr}$ & EN & $20,21,41$ \\
\hline 152 & Hydnum repandum L. : Fr. & + & $\mathrm{Mr}$ & & 18, SOMF \\
\hline 153 & Hygrocybe conica (Scop. : Fr.) P. Kumm. & + & $\mathrm{Hu}$ & & 31 \\
\hline 154 & H. persistens (Britzelm.) Singer & & $\mathrm{Hu}$ & & 41 \\
\hline 155 & Hygrophorus agathosmus (Fr.) Fr. & + & $\mathrm{Mr}$ & & 18 \\
\hline 156 & H. calophyllus P. Karst. & & $\mathrm{Mr}$ & & 23 \\
\hline 157 & H. chrysodon (Batsch : Fr.) Fr. & & $\mathrm{Mr}$ & & 23 \\
\hline 158 & H. eburneus (Bull. : Fr.) Fr. & & $\mathrm{Mr}$ & & 18 \\
\hline 159 & H. hypothejus (Fr. : Fr.) Fr. & & $\mathrm{Mr}$ & & 23 \\
\hline 160 & H. pudorinus (Fr.) Fr.* & & $\mathrm{Mr}$ & & SOMF \\
\hline 161 & Hypholoma capnoides (Fr.) P. Kumm. & + & LeS & & 18 \\
\hline 162 & H. fasciculare (Huds. : Fr.) Quél.* & & LeS & & SOMF \\
\hline 163 & H. sublateritium (Schaeff.) Quél. & + & LeS & & 18, SOMF \\
\hline 164 & Incrustoporia nivea (Jungh.) Ryvarden & & LeS & & 38 \\
\hline 165 & Inocybe bresadolae Massee & + & $\mathrm{Mr}$ & & 31 \\
\hline 166 & I. brunnea Quél. & + & $\mathrm{Mr}$ & & 31 \\
\hline 167 & I. hystrix (Fr.) P. Karst.* & & $\mathrm{Mr}$ & & SOMF \\
\hline 168 & I. lanuginosa (Bull. : Fr.) P. Kumm. & + & $\mathrm{Mr}$ & & 43 \\
\hline 169 & I. leucoblema Kühner & + & $\mathrm{Mr}$ & & 31 \\
\hline 170 & I. pisciodora Donadini \& Riousset* & + & $\mathrm{Mr}$ & & unpubl. \\
\hline 171 & I. praetervisa Quél. & & $\mathrm{Mr}$ & & 41 \\
\hline 172 & I. rimosa (Bull.) P. Kumm. & + & $\mathrm{Mr}$ & & 41 \\
\hline 173 & I. whitei (Berk. \& Broome) Sacc.* & + & $\mathrm{Mr}$ & & SOMF \\
\hline 174 & Inonotus hastifer Pouzar & & $\mathrm{LeS}$ & & 38 \\
\hline 175 & $\begin{array}{l}\text { Ischnoderma benzoinum (Wahlenb. : Fr.) } \\
\text { P. Karst. }\end{array}$ & + & LeS & & 10,36, SOA \\
\hline 176 & $\begin{array}{l}\text { Kuehneromyces mutabilis (Schaeff. : Fr.) } \\
\text { Singer \& A.H. Sm.* }\end{array}$ & & LeS & & SOMF \\
\hline 177 & Laccaria amethystina Cooke* & & $\mathrm{Mr}$ & & SOMF \\
\hline 178 & Lactarius aurantiacus (Pers.) Gray & + & $\mathrm{Mr}$ & & 18 \\
\hline 179 & L. blennius (Fr.) Fr. & + & $\mathrm{Mr}$ & & $17,18, \mathrm{SOMF}$ \\
\hline 180 & L. bresadolianus Singer* & + & $\mathrm{Mr}$ & & SOMF \\
\hline 181 & L. circellatus (Battarra) Fr. & & $\mathrm{Mr}$ & & 41 \\
\hline 182 & L. hepaticus Plowr. & + & $\mathrm{Mr}$ & & 43 \\
\hline 183 & L. picinus $\mathrm{Fr}$. & & $\mathrm{Mr}$ & & 41 \\
\hline 184 & L. piperatus (L. : Fr.) Pers. & + & $\mathrm{Mr}$ & & 3, SOMF \\
\hline 185 & L. rufus (Scop. : Fr.) Fr. & + & $\mathrm{Mr}$ & & 17 \\
\hline 186 & L. sanguifluus $\mathrm{Fr}$. & & $\mathrm{Mr}$ & & 41 \\
\hline 187 & L. scrobiculatus (Scop.) Fr.* & + & $\mathrm{Mr}$ & & SOMF \\
\hline 188 & L. semisanguifluus R. Heim \& Leclair & & $\mathrm{Mr}$ & & 41 \\
\hline 189 & L. subdulcis (Bull. : Fr.) Gray & + & $\mathrm{Mr}$ & & 17 \\
\hline 190 & L. volemus (Fr.) Fr.* & & $\mathrm{Mr}$ & & SOMF \\
\hline
\end{tabular}


Tab. 1 cont.

\begin{tabular}{|c|c|c|c|c|c|}
\hline 191 & Lentinus tigrinus (Bull. : Fr.) Fr.* & & LeS & & SOMF \\
\hline 192 & Lenzites warnieri Durieu \& Mont. & & LeS & NT & 21,38 \\
\hline 193 & Lepiota aspera (Pers. : Fr.) Quél. & & $\mathrm{Hu}$ & & 23, SOMF \\
\hline 194 & L. cristata (Bolton : Fr.) P. Kumm.* & & $\mathrm{Hu}$ & & SOMF \\
\hline 195 & Lepista nuda (Bull. : Fr.) Cooke & + & $\mathrm{Hu}$ & & 10 \\
\hline 196 & $\begin{array}{l}\text { Leucoagaricus leucothites (Vittad.) M.M. } \\
\text { Moser ex Bon* }\end{array}$ & + & $\mathrm{Hu}$ & & SOMF \\
\hline 197 & Lycoperdon atropurpureum Vittad. & + & $\mathrm{Hu}$ & & 10 \\
\hline 198 & L. decipiens Durieu \& Mont.* & + & $\mathrm{Hu}$ & & SOMF \\
\hline 199 & L. echinatum Pers. : Pers. & & $\mathrm{Hu}$ & & 41 \\
\hline 200 & L. ericetorum Pers. var. ericetorum & + & $\mathrm{Hu}$ & & $5,8, \mathrm{SOMF}$ \\
\hline 201 & L. marginatum Vittad. & + & $\mathrm{Hu}$ & & 9, SOMF \\
\hline 202 & L. molle Pers. : Pers.* & & $\mathrm{Hu}$ & & SOMF \\
\hline 203 & L. perlatum Pers. : Pers. & + & $\mathrm{Hu}$ & & 10 \\
\hline 204 & L. pyriforme Schaeff. : Pers.* & + & LeS & & $\begin{array}{l}\text { SOMF and } \\
\text { unpubl. }\end{array}$ \\
\hline 205 & L. spadiceum Schaeff. & & $\mathrm{Hu}$ & & 8, SOMF \\
\hline 206 & L. umbrinum Pers. : Pers. & + & $\mathrm{Hu}$ & & 10, SOMF \\
\hline 207 & Macrolepiota procera (Scop. : Fr.) Singer & + & $\mathrm{Hu}$ & & $\begin{array}{l}3,10,17,34, \\
\text { SOMF }\end{array}$ \\
\hline 208 & $\begin{array}{l}\text { Marasmiellus roseus (M.M. Moser) Kuyper } \\
\text { \& Noordel. }\end{array}$ & & $\mathrm{Hu}$ & & 41 \\
\hline 209 & Marasmius alliaceus (Jacq. : Fr.) Fr. & + & St, LeS & & 17,18, SOMF \\
\hline 210 & M. androsaceus (L. : Fr.) Fr. & + & Ad, LeS & & $10,17,43$ \\
\hline 211 & M. caryophylleus (Schaeff.) J. Schröt. & + & St & & 3 \\
\hline 212 & M. epiphyllus (Pers.) Fr. & + & St & & 10 \\
\hline 213 & M. insititius $\mathrm{Fr}$. & + & St & & 3 \\
\hline 214 & M. oreades (Bolton : Fr.) Fr. & + & $\mathrm{Mr}$ & & $\begin{array}{l}3,10,31 \\
\text { SOMF }\end{array}$ \\
\hline 215 & M. rotula (Scop. : Fr.) Fr. & + & LeS & & 3,10 \\
\hline 216 & M. scorodonius (Fr. : Fr.) Fr. & + & LeS, St & & 10 \\
\hline 217 & M. splachnoides (Hornem.) Fr. & + & Ad & & 3 \\
\hline 218 & M. wynnei Berk. \& Broome & + & $\begin{array}{l}\mathrm{Ad}, \mathrm{Fd}, \\
\mathrm{St}\end{array}$ & & 31 \\
\hline 219 & $\begin{array}{l}\text { Megacollybia platyphylla (Pers. : Fr.) Kotl. } \\
\text { \& Pouzar }\end{array}$ & + & LeS & & $10,41, \mathrm{SOMF}$ \\
\hline 220 & Melanoleuca bataillei Melençon & + & St & & 41 \\
\hline 221 & M. cognata (Fr.) Konrad \& Maubl. & + & St & & 31, SOMF \\
\hline 222 & M. exscissa (Fr.) Singer & + & St & & 10,41 \\
\hline 223 & M. grammopodia (Bull. : Fr.) Murrill & + & St & & 10, SOMF \\
\hline 224 & M. melaleuca (Pers. : Fr.) Murrill* & + & $\mathrm{Hu}$ & & SOMF \\
\hline 225 & M. oreina (Fr.) Kühner \& Maire & + & St & & $10,41, \mathrm{SOMF}$ \\
\hline 226 & $\begin{array}{l}\text { M. subalpina (Britzelm.) Brezinsky \& } \\
\text { Stangl }\end{array}$ & + & St & & $10,41, \mathrm{SOMF}$ \\
\hline 227 & Micromphale perforans (Hoffm. : Fr.) Gray & & Ad, St & & 23 \\
\hline 228 & Mycena crocata (Schrad.) Fr. & + & $\mathrm{Fd}, \mathrm{St}$ & & 18 \\
\hline 229 & M. epipterygia (Scop. : Fr.) Gray* & + & St, Ad & & SOMF \\
\hline 230 & M. galericulata (Scop. : Fr.) Schaeff. & + & LeS & & 17,18, SOMF \\
\hline 231 & M. leptocephala (Pers. : Fr.) Gillet* & + & $\mathrm{Hu}$ & & SOMF \\
\hline 232 & M. maculata P. Karst.* & + & LeS & & SOMF \\
\hline 233 & M. polygramma (Bull.) Gray & + & LeS & & 10, SOMF \\
\hline 234 & M. pura (Pers.) Sacc. & + & St & & $10,17,18$ \\
\hline 235 & M. renati Quél. & + & LeS & & 10,41 \\
\hline 236 & M. rosella (Fr.) P. Kumm. & & $\mathrm{Ad}, \mathrm{St}$ & & 23 \\
\hline 237 & M. vitilis (Fr.) Quél. & & LeS & & 41, SOMF \\
\hline 238 & Naucoria striatula P.D. Orton & & $\mathrm{Mr}$ & & 43 \\
\hline 239 & $\begin{array}{l}\text { Neolentinus lepideus (Fr. : Fr.) Redhead \& } \\
\text { Ginns }\end{array}$ & + & LeS & & 10 \\
\hline 240 & Onnia leporina (Fr.) H. Jahn & & LeS & & 38 \\
\hline 241 & O. tomentosa (Fr. : Fr.) P. Karst. & + & LeS & & $10,17,38$ \\
\hline 242 & $\begin{array}{l}\text { Oudemansiella mucida (Schrad. : Fr.) } \\
\text { Höhn. }\end{array}$ & & LeS, LeP & & 10 \\
\hline
\end{tabular}


Tab. 1 cont.

\begin{tabular}{|c|c|c|c|c|c|}
\hline 243 & $\begin{array}{l}\text { Panaeolus semiovatus (Sowerby. : Fr.) S. } \\
\text { Lundell \& Nannf. }\end{array}$ & & $\mathrm{C}$ & & $10, \mathrm{SOMF}$ \\
\hline 244 & Paxillus involutus (Batsch : Fr.) Fr. & + & $\mathrm{Mr}$ & & 2,17 \\
\hline 245 & Peniophora rufa (Pers. : Fr.) Boidin* & + & LeS & & unpubl. \\
\hline 246 & Phaeolus schweinitzii (Fr. : Fr.) Pat. & + & LeP & & $10,17,34$ \\
\hline 247 & Phellinus chrysoloma (Fr.) Donk & + & LeP, LeS & & 38 \\
\hline 248 & Ph. conchatus (Pers. : Fr.) Quél. & + & LeP, LeS & & 39 \\
\hline 249 & Ph. hartigii (Allesch. \& Schnabl) Pat. & + & LeP & & 10,39 \\
\hline 250 & Ph. igniarius (L. : Fr.) Quél. & + & LeP, LeS & & 39 \\
\hline 251 & $\begin{array}{l}\text { Ph. nigrolimitatus (Romell) Bourdot \& } \\
\text { Galzin }\end{array}$ & + & LeS & NT & 21,39 \\
\hline 252 & Ph. pini (Brot.) Bondartsev \& Singer & + & LeP & & $10,17,39$ \\
\hline 253 & Ph. torulosus (Pers.) Bourdot \& Galzin* & & LeP, LeS & & SOMF \\
\hline 254 & Pholiota astragalina (Fr.) Singer & + & LeS & & 43 \\
\hline 255 & Ph. highlandensis (Peck) A.H. Sm. \& Hesler & + & Carb & & 10,31 \\
\hline 256 & Ph. squarrosa (Weigel : Fr.) P. Kumm.* & + & LeP, LeS & & SOMF \\
\hline 257 & $\begin{array}{l}\text { Phylloporus pelletieri (Lév.) Quél. (Ph. } \\
\text { rhodoxanthus (Schwein. : Fr.) Bres.) }\end{array}$ & + & $\mathrm{Mr}$ & EN & $2,17,20,21$ \\
\hline 258 & Phyllotopsis nidulans (Pers. : Fr.) Singer & & LeS & NT & 20,21 \\
\hline 259 & Piptoporus betulinus (Bull. : Fr.) P. Karst. & + & LeP & & 10 \\
\hline 260 & Pleurotus cornucopiae (Paulet) Rolland* & & LeS & & SOMF \\
\hline 261 & P. ostreatus (Jacq. : Fr.) Quél. & + & LeS, LeP & & 10 \\
\hline 262 & Pluteus cervinus Schaeff. & + & LeS & & 17 \\
\hline 263 & Polyporus arcularius (Batsch : Fr.) Fr. & + & LeS & & 10,23 \\
\hline 264 & P. brumalis (Pers. : Fr.) Fr. & + & LeS & & 10,17 \\
\hline 265 & P. circinatus Fr. : Fr. & + & LeS & & 3 \\
\hline 266 & P. melanopus (Pers. : Fr.) Fr. & + & LeP, LeS & & $17,23,38$ \\
\hline 267 & P. neesii Fr. & + & LeS & & 3 \\
\hline 268 & P. squamosus (Huds. : Fr.) Fr. & + & LeP, LeS & & 10 \\
\hline 269 & P. varius (Pers. : Fr.) Fr. & + & LeS & & 17,41 \\
\hline 270 & Poria lindbladii (Berk.) Cooke & & LeS & & 38 \\
\hline 271 & Postia stipica (Pers. : Fr.) Jülich & & LeS & & 38 \\
\hline 272 & $\begin{array}{l}\text { Psathyrella ammophila (Durieu \& Lev.) } \\
\text { P.D. Orton* }\end{array}$ & & $\mathrm{Hu}$ & & SOMF \\
\hline 273 & P. gracilis (Fr. : Fr.) Quél. & + & $\mathrm{Hu}$ & & 3 \\
\hline 274 & P. pennata (Fr.) A. Pearson \& Dennis & + & Carb & & 10,41 \\
\hline 275 & $\begin{array}{l}\text { Pseudohydnum gelatinosum (Scop. : Fr.) P. } \\
\text { Karst. }\end{array}$ & + & LeS & & 17 \\
\hline 276 & Psilocybe montana (Pers. : Fr.) P. Kumm. & + & $\mathrm{Hu}$ & & 43, SOMF \\
\hline 277 & P. semilanceata (Fr.) P. Kumm. & + & $\mathrm{C}, \mathrm{Hu}$ & & 43 \\
\hline 278 & Pycnoporus cinnabarinus (Jacq. : Fr.) Fr. & + & LeS & & 10,38 \\
\hline 279 & Ramaria formosa (Pers.) Quél. & + & $\mathrm{Hu}$ & & 18,23, SOMF \\
\hline 280 & Rhizopogon roseolus (Corda) Th. Fr. & + & $\mathrm{Mr}$ & & 18,31, SOMF \\
\hline 281 & $\begin{array}{l}\text { Rhodocollybia butyracea (Bull.) Antonín \& } \\
\text { Noordel. }\end{array}$ & + & St & & 10,18, SOMF \\
\hline 282 & Rickenella swartzii (Fr. : Fr.) Kuyper & + & $\mathrm{Br}$ & & 43 \\
\hline 283 & $\begin{array}{l}\text { Rigidoporus ulmarius (Sowerby : Fr.) } \\
\text { Imazeki }\end{array}$ & & LeP & & 38 \\
\hline 284 & Russula adusta (Pers.) Fr. & + & $\mathrm{Mr}$ & & $3, \mathrm{SOMF}$ \\
\hline 285 & R. aeruginea Fr.* & + & $\mathrm{Mr}$ & & SOMF \\
\hline 286 & R. alutacea (Fr.) Fr.* & & $\mathrm{Mr}$ & & SOMF \\
\hline 287 & R. amethystina Quél. & + & $\mathrm{Mr}$ & EN & 21,41 \\
\hline 288 & R. aurea Pers. & + & $\mathrm{Mr}$ & & 18,41 \\
\hline 289 & R. badia Quél. & + & $\mathrm{Mr}$ & & 41 \\
\hline 290 & R. caerulea (Pers.) Fr. & + & $\mathrm{Mr}$ & & 3 \\
\hline 291 & R. cyanoxantha (Schaeff.) Fr. & + & $\mathrm{Mr}$ & & 17,18, SOMF \\
\hline 292 & R. delica Fr. & + & $\mathrm{Mr}$ & & 18, SOMF \\
\hline 293 & R. foetens (Pers. : Fr.) Fr. & + & $\mathrm{Mr}$ & & 18 \\
\hline 294 & R. grata Britzelm.* & + & $\mathrm{Mr}$ & & SOMF \\
\hline 295 & R. grisea (Batsch) Fr. & + & $\mathrm{Mr}$ & & 41 \\
\hline 296 & R. integra (L.) Fr. & + & $\mathrm{Mr}$ & & 18,41 \\
\hline
\end{tabular}


Tab. 1 cont.

\begin{tabular}{|c|c|c|c|c|c|}
\hline 297 & R. melliolens Quél. & + & $\mathrm{Mr}$ & & 41 \\
\hline 298 & R. mustelina Fr.* & & $\mathrm{Mr}$ & & SOMF \\
\hline 299 & R. paludosa Britzelm. & + & $\mathrm{Mr}$ & & 18,41 \\
\hline 300 & R. puellaris Fr. & & $\mathrm{Mr}$ & & 41 \\
\hline 301 & R. rosea Pers. & & $\mathrm{Mr}$ & & 41, SOMF \\
\hline 302 & R. sanguinea (Bull.) Fr. & + & $\mathrm{Mr}$ & & 43 \\
\hline 303 & R. xerampelina (Schaeff.) Fr.* & & $\mathrm{Mr}$ & & SOMF \\
\hline 304 & Sarcodon imbricatus (L. : Fr.) P. Karst. & + & $\mathrm{Mr}$ & & 10; SOMF \\
\hline 305 & S. leucopus (Pers.) Maas Geest. \& Nannf. & + & $\mathrm{Mr}$ & CR & $\begin{array}{l}\text { 21, 23, 41, } \\
\text { SOMF }\end{array}$ \\
\hline 306 & Schizopora paradoxa (Schrad. : Fr.) Donk* & + & LeS & & unpubl. \\
\hline 307 & $\begin{array}{l}\text { Scleroderma verrucosum (Bull. : Pers.) } \\
\text { Pers.* }\end{array}$ & + & $\mathrm{Hu}$ & & SOMF \\
\hline 308 & $\begin{array}{l}\text { Skeletocutis amorpha (Fr. : Fr.) Kotl. \& } \\
\text { Pouzar }\end{array}$ & & LeS & & 38 \\
\hline 309 & Sparassis crispa (Wulfen : Fr.) Fr. & + & $\mathrm{Mr}$ & EN & 21,35 \\
\hline 310 & Stereum hirsutum (Willd. : Fr.) Gray & + & LeS & & $3,17,28$ \\
\hline 311 & S. sanguinolentum (Alb. \& Schwein.) Fr. & + & LeS & & 17,38 \\
\hline 312 & S. spadiceum (Pers.) Quél. & + & LeS & & 3 \\
\hline 313 & Strobilomyces strobilaceus (Scop. : Fr.) Berk. & & $\mathrm{Mr}$ & $\mathrm{VU}$ & $\begin{array}{l}1,2,20,21, \\
41, \text { SOMF }\end{array}$ \\
\hline 314 & Strobilurus esculentus (Wulfen : Fr.) Singer & + & $\mathrm{S}$ & & $10,18,41$ \\
\hline 315 & $\begin{array}{l}\text { S. stephanocystis (Kühner \& Romagn. ex } \\
\text { Hora) Singer }\end{array}$ & + & $\mathrm{S}$ & & 10,18, SOMF \\
\hline 316 & S. tenacellus (Pers. : Fr.) Singer & + & $\mathrm{S}$ & & $10, \mathrm{SOMF}$ \\
\hline 317 & Stropharia aeruginosa (Curtis : Fr.) Quél. & + & $\mathrm{Hu}$ & & 18,23 \\
\hline 318 & S. coronilla (Bull. : Fr.) Fr. & + & $\mathrm{Hu}$ & & 10 \\
\hline 319 & S. semiglobata (Batsch : Fr.) Quél. & + & $\mathrm{C}$ & & 17,28 \\
\hline 320 & S. squamosa (Pers. : Fr.) Quél. & + & LeS & & 10 \\
\hline 321 & Suillus bovinus (L. : Fr.) Roussel & + & $\mathrm{Mr}$ & & 2,3 \\
\hline 322 & S. granulatus (L. : Fr.) Roussel & + & $\mathrm{Mr}$ & & 2,34 \\
\hline 323 & S. grevillei (Klotzsch) Singer & & $\mathrm{Mr}$ & & $2,10, \mathrm{SOMF}$ \\
\hline 324 & S. luteus (L. : Fr.) Roussel & + & $\mathrm{Mr}$ & & 2,43 \\
\hline 325 & $\begin{array}{l}\text { S. sibiricus (Singer) Singer subsp. helveticus } \\
\text { Singer }\end{array}$ & + & $\mathrm{Mr}$ & EN & $\begin{array}{l}1,2,17,18 \\
20,21,40 \\
\text { SOMF }\end{array}$ \\
\hline 326 & $\begin{array}{l}\text { S. variegatus (Schwartz : Fr.) Richon \& } \\
\text { Roze }\end{array}$ & + & $\mathrm{Mr}$ & & $\begin{array}{l}\text { 1,2,17, } \\
\text { SOMF }\end{array}$ \\
\hline 327 & Thelephora palmata (Scop. : Fr.) Fr.* & + & $\mathrm{Mr}$ & & SOMF \\
\hline 328 & Tomentella crinalis (Fr.) M.J. Larsen & + & LeS & & 41 \\
\hline 329 & Trametes gibbosa (Pers. : Fr.) Fr. & & LeS & & 38 \\
\hline 330 & T. hirsuta (Wulfen : Fr.) Pilát & + & LeS & & 17,38 \\
\hline 331 & T. pubescens (Schumach. : Fr.) Pilát & & LeS & & 38 \\
\hline 332 & T. versicolor (L. : Fr.) Lloyd & + & LeS & & $10,17,38$ \\
\hline 333 & $\begin{array}{l}\text { Trichaptum abietinum (Dicks. : Fr.) } \\
\text { Ryvarden }\end{array}$ & + & LeS & & 10,17 \\
\hline 334 & T. fuscoviolaceum (Ehrenb. : Fr.) Ryvarden & & LeS & & 38 \\
\hline 335 & Tricholoma albobrunneum (Pers.) P. Kumm. & & $\mathrm{Mr}$ & & 23, SOMF \\
\hline 336 & T. atrosquamosum (Chevall.) Sacc. & + & $\mathrm{Mr}$ & & 41 \\
\hline 337 & T. colossus (Fr.) Quél. & + & $\mathrm{Mr}$ & CR & $10,20,21,41$ \\
\hline 338 & T. flavovirens (Pers. : Fr.) S. Lundell & & $\mathrm{Mr}$ & & 23 \\
\hline 339 & T. imbricatum (Fr.) P. Kumm.* & + & $\mathrm{Mr}$ & & SOMF \\
\hline 340 & T. portentosum (Fr. : Fr.) Quél. & & $\mathrm{Mr}$ & & 23 \\
\hline 341 & T. saponaceum (Fr. : Fr.) P. Kumm. & + & $\mathrm{Mr}$ & & 17,18, SOMF \\
\hline 342 & T. terreum (Schaeff. : Fr.) Quél.* & & $\mathrm{Mr}$ & & SOMF \\
\hline 343 & $\begin{array}{l}\text { Tricholomopsis rutilans (Schaeff. : Fr.) } \\
\text { Singer }\end{array}$ & + & LeS & & $10,18, \mathrm{SOMF}$ \\
\hline 344 & $\begin{array}{l}\text { Tylopilus pseudoscaber Secr. ex A.H. Smith } \\
\& \text { Thiers (Porphyrellus porphyrosporus (Fr. } \\
\text { \& Hök.) E.-J. Gilbert) }\end{array}$ & + & $\mathrm{Mr}$ & $\mathrm{VU}$ & $\begin{array}{l}\text { 1,2,10,17, } \\
20,21, \text { SOMF }\end{array}$ \\
\hline 345 & Tyromyces caesius (Schrad. : Fr.) Murrill & & LeS & & 38 \\
\hline 346 & T. subcaesius A. David & + & LeS & & 10 \\
\hline
\end{tabular}




\begin{tabular}{|c|l|l|l|l|l|}
\hline 347 & T. tephroleucus (Fr. : Fr.) Donk & & LeS & 38 \\
\hline 348 & Vascellum pratense (Pers. : Pers.) Kreisel & & Hu & 6 \\
\hline 349 & $\begin{array}{l}\text { Xeromphalina campanella (Batsch : Fr.) } \\
\text { Maire }\end{array}$ & + & LeS & 17,18, SOMF \\
\hline 350 & Xerula radicata (Relhan : Fr.) Fr. & + & LeS & $17,18,43$ \\
\hline
\end{tabular}

Explanations: *New records for the Pirin Mts; **National Park Pirin; ***Ecological-trophic groups: Ad - needle-debris saprotrophs, Fd - leaf-debris saprotrophs, $\mathbf{S}$ - cone saprotrophs, $\mathbf{S t}$ - litter saprotrophs, $\mathbf{H u}$ - humus saprotrophs, LeS - wood saprotrophs, $\mathbf{B r}$ - moss saprotrophs, $\mathbf{C}$ - coprotrophs, $\mathbf{M}$ - fungal saprotrophs, Carb - carbotrophs, Mr - mycorrhizal fungi, LeP - wood parasites; ****IUCN categories: CR - Critically Endangered, EN - Endangered, VU - Vulnerable, NT - Near Threatened; *****For the assigned numbers of literature sources, see the references rubric

List of synonyms: Agaricus macrosporus (F.H Møller \& Jul. Schäff.) Pilát. = Agaricus albertii, Anellaria semiovata (Sowerby. : Fr.) A. Pearson \& Dennis = Panaeolus semiovatus, Boletus piperatus Bull. : Fr. = Chalciporus piperatus, Caldesiella ferruginosa (Fr.) Sacc. = Tomentella crinalis, Calvatia excipuliformis (Scop. : Pers.) Perdeck = Handkea excipuliformis, Calvatia utriformis (Bull. : Pers.) Jaap = Handkea utriformis, Clavaria formosa Pers. = Ramaria formosa, Clavaria ligula Schaeff. : Fr. = Clavariadelphus ligula, Clavaria rugosa Bull. = Clavulina rugosa, Clitocybe infundibuliformis (Schaeff.) Fr. = Clitocybe gibba, Collybia butyracea (Bull. : Fr.) P. Kumm. = Rhodocollybia butyracea, Collybia peronata (Bolton : Fr.) P. Kumm. = Gymnopus peronatus, Conocybe magnicapitata P.D. Orton = Conocybe juniana, Coriolopsis trogii (Berk.) Domański = Funalia trogii, Coriolus hirsutus (Wulf. : Fr.) Pat. = Trametes hirsuta, Crepidotus variabilis (Pers. : Fr.) P. Kumm. = Claudopus variabilis, Cystoderma terrei (Berk. \& Broome) Harmaja = Cystoderma cinnabarinum, Daedalea biennis (Bull.) Fr. = Abortiporus biennis, Dasyscyphus bicolor (Bull. : Fr.) Fuckel = Lachnum bicolor, Dasyscyphus brevipilus Le $\mathrm{Gal}=$ Lachnum brevipilosum, Dasyscyphus clandestinus (Bull. : Fr.) Fuckel = Brunnipila clandestine, Dasyscyphus mollissimus (Lasch) Nannf. = Lasiobelonium mollissimum, Dasyscyphus nidulus (J.C. Schmidt \& Kunze) Massee = Lasiobelonium nidulum, Dasyscyphus virgineus (Batsch : Fr.) Gray = Lachnum virgineum, Deconica physaloides (Bull.) P. Karst. = Psilocybe montana, Dermocybe crocea (Schaeff.) M.M. Moser = Cortinarius croceus, Dermocybe punicea (P.D. Orton) M.M. Moser = Cortinarius sanguineus, Diplomitoporus lindbladii (Berk.) Gilb. \& Ryvarden = Poria lindbladii, Discina perlata (Fr. : Fr.) Fr. = Discina ancilis, Echinoderma asperum (Pers. : Fr.) Bon $=$ Lepiota aspera, Fomes pinicola (Sw.) Fr. = Fomitopsis pinicola, Galerina rubiginosa (Pers.) Kühner = Galerina vittiformis, Ganoderma atkinsonii $\mathrm{H}$. Jahn, Kotl. \& Pouzar = Ganoderma carnosum, Geastrum nanum Pers. = Geastrum schmidelii, Geastrum sessile (Sowerby) Pouzar = Geastrum fimbriatum, Gomphidius rutilus (Schaeff. : Fr.) S. Lundell = Chroogomphus rutilus, Gyrocephalus rufus (Jacq.) Bref. = Guepinia helvelloides, Helotium clavus (Alb. \& Schwein.) Gillet = Cudoniella clavus, Hemitrichia vesparium (Batsch) T. Macbr. = Metatrichia vesparium, Herpotrichia nigra R. Hartig = Herpotrichia juniperi, Hirschioporus abietinus (Dicks. : Fr.) Donk = Trichaptum abietinum, Hydnum imbricatum L. : Fr. = Sarcodon imbricatus, Hygrocybe acutoconica (Clem.) Singer = Hygrocybe persistens, Inocybe fastigiata (Schaeff. : Fr.) Quél. = Inocybe rimosa, Inocybe longicystis G.F. Atk. = Inocybe lanuginosa, Inocybe pudica Kühner = Inocybe whitei, Lachnella oblongospora (G.G.Hahn. \& Ayers) Seaver = Dasyscyphus oblongosporus, Lactarius mitissimus (Fr.) Fr. = Lactarius aurantiacus, Lentinus lepideus (Fr. : Fr.) Fr. = Neolentinus lepideus, Lenzites abietina (Bull.) Fr. = Gloeophyllum abietinum, Lenzites sepiaria (Wulfen : Fr.) Fr. = Gloeophyllum sepiarium, Lepiota acutesquamosa (Weinm.) P. Kumm. = Lepiota aspera, Leucoporus arcularius (Batsch : Fr.) Quél. = Polyporus arcularius, Marasmius perforans (Hoffm. : Fr.) Fr. = Micromphale perforans, Macrolepiota rhacodes (Vittad.) Singer = Chlorophyllum rhacodes, Melanoleuca cinerascens D.A. Reid = Melanoleuca exscissa, Morchella conica Pers. = Morchella vulgaris, Mycena gracilis (Quél.) Kühner = Hemimycena gracilis, Mycena chlorinella (J.E. Lange) Singer = Mycena leptocephala, Mycena swartzii (Fr. : Fr.) A.H. Sm. = Rickenella swartzii, Nyctalis parasitica (Bull. : Fr.) Fr. = Asrerophora parasitica, Oligoporus caesius (Schrad. : Fr.) Gilb. \& Ryvarden = Tyromyces caesius, Oligoporus stipticus (Pers. : Fr.) Gilb. \& Ryvarden = Postia stipica, Oligoporus tephroleucus (Fr. : Fr.) Gilb. \& Ryvarden = Tyromyces tephroleucus, Omphalina chrysophylla (Fr.) Murrill = Chrysomphalina chrysophylla, Omphalina rosella (M.M. Moser) M.M. Moser = Marasmiellus roseus, Oudemansiella platyphylla (Pers. : Fr.) M.M. Moser = Megacollybia platyphylla, Oudemansiella radicata (Relhan : Fr.) Singer = Xerula radicata, Peziza anthracophila Dennis = Peziza echinospora, Peziza lilacina (Boud.) Sacc. \& Traverso = Peziza moseri, Polystictus perennis (L. : Fr.) Fr. = Coltricia perennis, Psaliota arvensis (Schaeff. : Fr.) Gillet = Agaricus arvensis, Phialea cyathoidea (Bull.) Gillet = Crocicreas cyathoideum, Pholiota carbonaria (Batsch $:$ Fr.) Singer = Pholiota highlandensis, Pholiotina blattaria (Fr.) Fayod = Conocybe blattaria, Polyporus nummularius (Bull. : Fr.) Pers. = Polyporus varius, Polyporus perennis (L. : Fr.) Fr. = Coltricia perennis, Porphyrellus pseudoscaber Secr. ex Singer = Porphyrellus porphyrosporus, Psilocybe physaloides (Bull.) Quél. = Psilocybe montana, Rhytisma empetri Fr. = Duplicaria empetri, Russula aurata (With.) Fr. = Russula aurea, Russula laurocerasi Melzer = Russula grata, Skeletocutis nivea (Jungh.) Jean Keller = Incrustoporia nivea, Spongipellis borealis (Fr. : Fr.) Pat. = Climacocystis borealis, Strobilomyces floccopus (Vahl : Fr.) P. Karst. = Strobilomyces strobilaceus, Stropharia semiglobata (Batsch : Fr.) Quél. var. stercoraria (Bull. : Fr.) K.-D. Jahnke = Stropharia semiglobata, Trametes trogii Berk. = Funalia trogii, Tremiscus helvelloides (DC.) Donk = Guepinia helvelloides, Trichaptum hollii (J.C. Schmidt) Rea = Trichaptum fuscoviolaceum, Trichaster melanocephalus Czern. = Geastrum melanocephalum, Vascellum depressum (Bonord.) F. Smarda = Vascellum pretense, Xerocomus chrysenteron (Bull.) Quél. = Boletus chrysenteron, Xerocomus spadiceus (Fr.) Quél. = Boletus ferrugineus, Xerocomus subtomentosus (L. : Fr.) Fr. = Boletus subtomentosus 


\section{RESULTS}

Species composition. The current knowledge on the macroscopic fungi, both for ascomycetes and basidiomycetes, of the Pirin Mts comprises 350 species (Tab. 1). For the ascomycetes, 7 orders, 17 families, 26 genera, and 34 species are established. Regarding basidiomycetes, there are found 10 orders, 45 families, 128 genera, and 316 species.

The families with highest species diversity are: Tricholomataceae (54 species or $15.4 \%$ of the total species number), Russulaceae (33 species, $9.4 \%$ ), Polyporaceae (30 species, 8.6\%), Cortinariaceae and Marasmiaceae (19species, 5.4\%), Lycoperdaceae (15 species, $4.3 \%$ ), Strophariaceae and Boletaceae (13 species, $3.7 \%$ ), Agaricaceae and Hymenochaetaceae (11 species, $3.1 \%$ ). Regarding the species diversity, the richest genera are: Russula (20 species), Lactarius (13), Lycoperdon, Marasmius, and Mycena (10), Inocybe and Boletus (9), Cortinarius and Tricholoma (8), Clitocybe, Melanoleuca, Phellinus, and Polyporus (7), Coprinus, Amanita, Hygrophorus, and Suillus (6).

The amount of 263 species (75.1\% of the total species number in the mountain) was estimated for the Pirin National Park so far. Twenty-six species of ascomycetes and 237 species of basidiomycetes were established (Tab. 1).

The mycological investigations of the reserves in Pirin Mts are still in an initial stage. Scanty studies were conducted only in Bayuvi Doupki-Dzhindzhiritsa Reserve. There are no records in the literature on the macroscopic fungi in the Yulen Resrve. In Bayuvi Doupki-Dzhindzhiritsa Reserve, 58 species were established (16.6\% of the total species number in the mountain and $22 \%$ from the species, found in Pirin National Park), as follows: Amanita vaginata, Boletus chrysenteron, B. edulis, B. erythropus, B. ferrugineus, B. luridus, B. pinophilus, B. piperatus, Cantharellus cibarius, Chroogomphus rutilus, Clitocybe clavipes, Coprinus laanii, C. radians, C. xanthothrix, Cortinarius croceus, Cudoniella clavus, Fomitopsis pinicola, Galerina marginata, Gloeophyllum abietinum, G. sepiarium, Gyroporus cyanescens, Heterobasidion annosum, Hydnum repandum, Hygrophorus agathosmus, H. eburneus, Hypholoma capnoides, H. sublateritium, Inocybe pisciodora, Lactarius aurantiacus, L. blennius, Lycoperdon pyriforme, Marasmius alliaceus, Mycena crocata, M. galericulata, M. pura, Peniophora rufa, Phellinus conchatus, Ph. nigrolimitatus, Ramaria formosa, Rhizopogon roseolus, Rhodocollybia butyracea, Russula adusta, $R$. aurea, $R$. badia, $R$. cyanoxantha, $R$. delica, $R$. foetens, $R$. integra, R. paludosa, Strobilurus esculentus, S. stephanocystis, Stropharia aeruginosa, $S$. semiglobata var. stercoraria, Suillus sibiricus, Tricholoma saponaceum, Tricholomopsis rutilans, Xeromphalina campanella, and Xerula radicata. These data are still preliminary and do not represent the potential diversity of the Pirin Mts reserves.

During the field investigations as well as after the inventory of SOMF, 60 species were found as new for the Pirin Mts, namely Gyromitra esculenta, Helvella acetabulum, H. lacunosa, Aleuria aurantia, Sarcoscypha coccinea, Caloscypha fulgens, Agaricus augustus, A. campestris, Lepiota cristata, Leucoagaricus leucothites, Bolbitius vitellinus, Hebeloma circinans, H. edurum, Coprinus atramentarius, C. laanii, C. radians, C. xanthothrix, Psathyrella ammophila, Cortinarius lustratus, C. venetus, Inocybe hystrix, I. pisciodora, I. whitei, Claudopus variabilis, Clitopilus prunulus, Laccaria amethystina, Handkea excipuliformis, H. utriformis, Lycoperdon decipiens, L. molle, L. pyriforme, Pleurotus cornucopiae, Amanita muscaria, Hypholoma fasciculare, Kuehneromyces mutabilis, Pholiota squarrosa, Clitocybe suaveolens, Hygrophorus pudorinus, Melanoleuca melaleuca, Mycena epipterygia, M. leptocephala, M. maculata, Tricholoma imbricatum, T. terreum, Scle- 
roderma verrucosum, Phellinus torulosus, Schizopora paradoxa, Geastrum pectinatum, Lentinus tigrinus, Peniophora rufa, Lactarius bresadolianus, L. scrobiculatus, L. volemus, Russula aenuginea, $R$. alutacea, $R$. grata, $R$ mustelina, $\boldsymbol{R}$ xerampelina, Hydnellum ferngineum, and Thelephora palmata. Inocybe pisciodora is a new record for Bulgaria.

In order to establish the actual fungal diversity in the Pirin Mts, purposeful and systematic mycological studies are needed. In the course of these studies, numerous new records for the mountain and the country are expected.

Ecological-trophic structure. Twelve ecological-trophic groups of fungi were determined in the plant communities of Pirin Mts (Tab. 1). Analysis of the published data showed that mycorrhizal fungi represented the highest number of species (33\% of the total species number), followed by wood saprotrophs (32\%), humus saprotrophs $(19 \%)$, and litter saprotrophs $(8 \%)$. The affiliation of the macromycetes to the respective groups is as follows: needle-debris saprotrophs (Ad) - 9 species, leaf-debris saprotrophs (Fd) -9 , cone saprotrophs $(\mathrm{S})-6$, litter saprotrophs (St) 29 , humus saprotrophs $(\mathrm{Hu})-66$, wood saprotrophs $(\mathrm{LeS})-111$, moss saprotrophs (Br) -3 , coprotrophs $(\mathrm{C})-5$, fungal saprotrophs $(\mathrm{M})-1$, carbotrophs (Carb) -5 , mycorrhizal fungi (Mr) - 114, and wood parasites (LeP) - 19 (Fig. 2).

Among the biotrophs, there are species with high economic importance: Armillaria mellea (on variety of trees), Phellinus conchatus (on Salix), Ph. hartigii (on Abies alba), Ph. igniarius (on Salix), Ph. pini (on Pinus heldreihii, P. mugo), Fomitopsis pinicola (on Picea abies, Abies alba, Pinus peuce), Heterobasidion annosum (on Picea abies, Abies alba), Phaeolus schweinitzii (on Picea abies), etc.

Economically important edible fungi and species of conservation significance. On the studied territory, 9 species of economic importance were found: Agaricus arvensis, A. campestris, A. silvaticus, Boletus edulis, B. pinophillus, Cantharellus cibarius, Lepista nuda, Macrolepiota procera, Marasmius oreades.

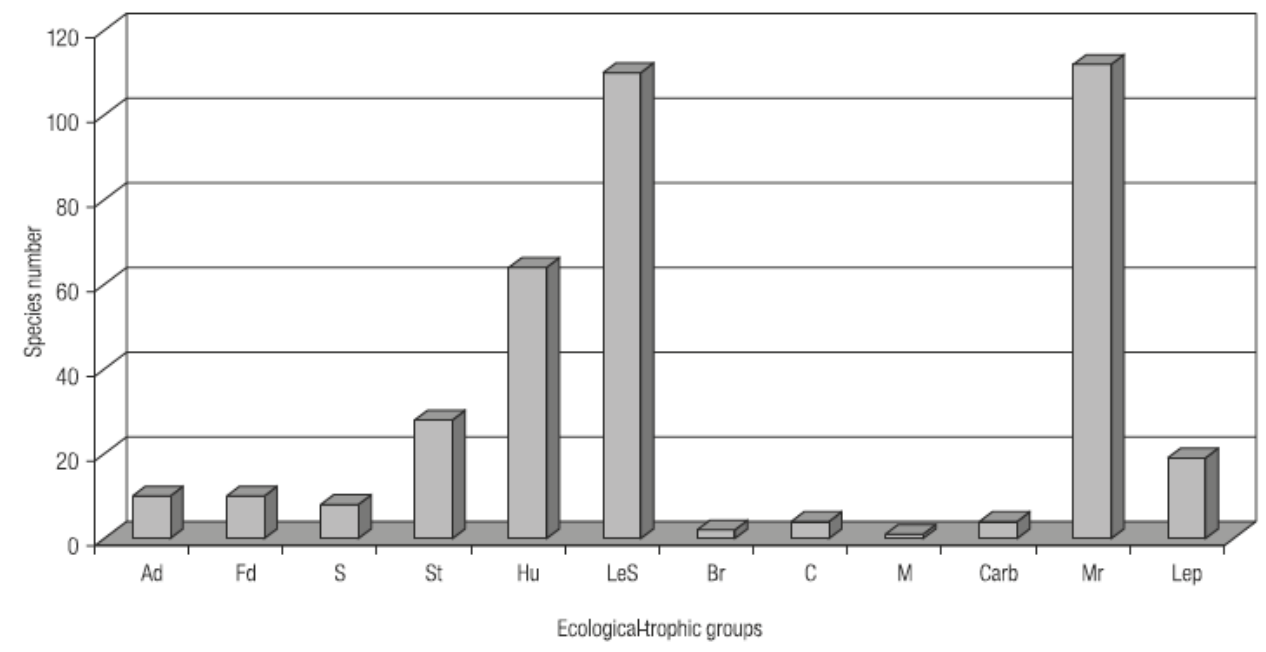

Fig. 2. Arrangement of fungal species in ecological-trophic groups.

Abbreviations: Ad - needle-debris saprotrophs, $\mathrm{Fd}$ - leaf-debris saprotrophs, $\mathrm{S}$ - cone saprotrophs, $\mathrm{St}$ litter saprotrophs, $\mathrm{Hu}$ - humus saprotrophs, LeS - wood saprotrophs, $\mathrm{Br}$ - moss saprotrophs, $\mathrm{C}$ - coprotrophs, M - fungal saprotrophs, Carb - carbotrophs, Mr - mycorrhizal fungi, LeP - wood parasites. 
In the last few years, the trade in economically important mushroom species has been gaining more attention and, thereupon, a huge progress. Such valuable edible species are Boletus edulis, B. pinophillus, Cantharellus cibarius, Marasmius oreades, etc. However, the activity of gathering and buying up mushrooms has lots of disadvantages, the most important of which is the lack of a normative base for the wild mushrooms in Bulgaria, regulating this process.

Our analysis arrived at the conclusion that the populations of Boletus edulis, $B$. pinophilus, and Cantharellus cibarius needed further restrictive regime of collection. The gathering of these mushrooms should be permited only in the areas of resorts, chalets, and along the mountain roads.

In the Pirin Mts, there are distributed the following 25 species of conservation significance: Critically Endangered (CR) - 4 species (Elaphomyces granulatus, Tricholoma colossus, Gyrodon lividus, and Sarcodon leucopus), Endangered (EN) - 9 species (Endoptychum agaricoides, Clitocybe vermicularis, Phylloporus pelletieri, Suillus sibiricus, Sparassis crispa, Auriscalpium vulgare, Russula amethystina, Hydnellum suaveolens, and Guepinia helvelloides), Vulnerable (VU) - 7 species (Discina ancilis, Otidea onotica, Urnula craterium, Strobilomyces strobilaceus, Tylopilus pseudoscaber, Geastrum melanocephalum, and Clavariadelphus ligula), Near Threatened (NT) 5 species (Spathularia flavida, Morchella elata, Phyllotopsis nidulans, Phellinus nigrolimitatus, and Lenzites warnieri).

\section{REFERENCES}

Assyov B. 2004. New data about Boletales in Bulgaria. Mycol. Balcan. 1: 85-88. [1]

Assyov B., Denchev C. M. 2004. Preliminary checklist of Boletales s. str. in Bulgaria. Mycol. Balcan. 1: 195-208. [2]

Burzakov B. 1928. [Contribution to the study of the fungus flora of Bulgaria]. God. Sofiisk. Univ. Fiz.Mat. Fak. 24(2-3): 1-18 (in Bulgarian). [3]

Burzakov B. 1932. [Two truffle species and a few fungi new for Bulgaria]. Izv. Bulg. Bot. Druzh. 5: 84-86 (in Bulgarian). [4]

Chalukov V. G. 1978. [New taxa and chorological data on Bulgarian flora]. Fitologiya 9: 70-72 (in Bulgarian). [5]

Chalukov V. 1982. [New data on the species composition and distribution of class Gasteromycetes in Bulgaria]. Fitologiya 19: 83-84 (in Bulgarian). [6]

Chalukov V. 1984. [Ecologic and taxonomic studies on Bovista in Bulgaria]. (In:) V. Velchev, (ed.). Modern theoretical and applied aspects of the plant ecology, Plovdiv, 10-13 October 1983: 298-305.

Bulgarian Academy of Sciences Publishing House, Sofia (in Bulgarian). [7]

Ch alu kov V. 1985. [Genus Lycoperdon Pers. in Bulgaria]. Fitologiya 28: 41-51 (in Bulgarian). [8]

Chaluk ov V. 1987. [New for Bulgaria taxa of Gasteromycetes]. Fitologiya 33: 70 (in Bulgarian). [9]

Dimcheva M. D., Stoichev G. T. 1987. [Trophic characteristic of macromycetes established in Pirin mountain]. (In:) B. Kuzmanov (ed.). Proceedings of the $4^{\text {th }}$ National Conference of Botany, Sofia, 1987, 1: 220-226. Bulgarian Academy of Sciences Publishing House, Sofia (in Bulgarian). [10]

Dimitrova E. G. 1994. A contribution to the study of the Discomycetes fungi in Bulgaria. II. Fitologiya 47: 74-77. [11]

Dimitrova E. 1997. Revision notes on the discomycetous fungi from Helotiales in Bulgaria. Phytol. Balcan. 3 (2-3): 211-215. [12]

Dimitrova E. 1998. Genus Crocicreas Fr. in Bulgaria. Phytol. Balcan. 4 (3): 131-139. [13]

Dimitrova E. 1999. Discomycetous fungi found in Bulgaria on needles, cones and twigs of conifers. Phytol. Balcan. 5 (1): 137-144. [14]

Dimit rov a E. 2000. A taxonomic study of Hyaloscyphaceae in Bulgaria. II. Dasyscyphus, Lachnum and Trichopezizella. Phytol. Balcan. 6 (1): 133-145. [15]

Dörfelt H. 1977. Zur Taxonomie, Verbreitung und Ökologie des filzigen Schmierlings Chroogomphus helveticus (Sing.) Moser. Feddes Repert. 88 (4): 273-285 + Tabs I-II. [16] 
Dörfelt H., Müsch F. 1987. Mykologische Studien in Pinus peuce-Waldern der Volksrepublik Bulgarien. Feddes Repert. 98 (7-8): 419-431. [17]

Drumeva-Dimcheva M., Gyosheva-Bogoeva M. 1993. [Macromycetes in Bulgaria]. (In:) M. Sakalian (ed.). The National Biological Diversity Conservation Strategy, 1: 1-34. Sofia (in Bulgarian). [18]

Gyosheva M. 2000. New and rare macromycetous taxa to Bulgaria. Phytol. Balcan. 6 (2-3): 283-288. [19]

Gyosheva M., Fakirova V., Denchev C. 2000. Red list and threat status of Bulgarian macromycetes. Hist. Nat. Bulg. 11: 139-145. [20]

Gyosheva M. M., Denchev C. M., Dimitrova E. G., Assyov B., Petrova R. D., Stoichev G. T. 2006. Red List of fungi in Bulgaria. Mycol. Balcan. 3: 81-87. [21]

Hin k ova Ts. 1961. [Materials on the fungus flora of Bulgaria]. Izv. Bot. Inst. (Sofia) 8: 251-259 (in Bulgarian). [22]

Hin k ov a Ts. 1965. [Contribution to the fungus flora of Bulgaria]. God. Sofiisk. Univ. Biol. Fak., 2. Bot., Mikrobiol., Fiziol. Biohim. Rast. 58 (2) [1963/1964]: 95-105 (in Bulgarian). [23]

IUCN 2001. IUCN Red List categories and criteria: Version 3.1. IUCN Species Survival Commission, IUCN, Gland, Switzerland and Cambridge, UK. [24]

IUCN 2003a. Guidelines for application of IUCN Red List categories at regional levels: Version 3.0. IUCN species survival Commission, IUCN, Gland, Switzerland and Cambridge, UK. [25]

IUCN 2003b. Guidelines for using the IUCN Red List categories and criteria. Standards and Petitions Subcommittee of the IUCN SSC Red List Programme Committee, IUCN, Gland, Switzerland and Cambridge, UK. [26]

Jordan ov D. (ed.) 1966. [Flora of People's Republic of Bulgaria]. Vol. 3. Bulgarian Academy of Sciences Publishing House, Sofia (in Bulgarian). [27]

Kr e is el H. 1959. Beitrage zur Pilzflora Bulgariens. Feddes Repert. 62 (1): 34-43. [28]

Kirk P. M., Cannon P. F., David J. C., Stalpers J. A. (eds) 2001. Dictionary of the fungi. $9^{\text {th }}$ ed. CAB International, Oxon. [29]

Kir k P. M. et al. 2004. Authors of fungal names. CABI Bioscience, Wallingford. Electronic version: $h t t p: / /$ www.speciesfungorum.org/AuthorsOfFungalNames.htm. [30]

Kuth an J., Kotlaba F. 1989. Makromyzeten der bulgarischen Schwarzmeerküste und einiger Orte im landesinnern Bulgariens. Acta Mus. Nat. Pragae, Ser. B 44 (3-4) [1988]: 137-243 + Photos 1-2 \& Tabs I/1-XVI/2. [31]

Lacheva M. N., Stoichev G. T. 2004. New species of the genus Agaricus (Agaricaceae) for Bulgaria. Mycol. Balcan. 1: 35-40. [32]

La Porta N., A postolov K., Kornonen K. 1998. Intersterility groups of Heterobasidion annosum and their host specificity in Bulgaria. Eur. J. Forest. Pathol. 28: 1-9. [33]

Pilát A. 1926. Nekolik poznámek k mycologickym pomérum Bulharska. Mycologia 3: 24-30. [34]

St oich ev G. T. 1981. [New taxa for the Bulgarian fungus flora]. Nauchni Trudove Vissh Selskost. Inst. "Vasil Kolarov" 26 (4): 105-107 (in Bulgarian). [35]

Stoichev G. T. 1987a. [Seven species of Polyporaceae new for Bulgaria]. (In:) B. Kuzmanov (ed.). Proceedings of the $4^{\text {th }}$ National Conference of Botany, Sofia, 1987, 1: 208-215. Publishing House of Bulgarian Academy of Sciences, Sofia (in Bulgarian). [36]

Stoich ev G. T. 1987b. [New chorological data for Polyporaceae in Bulgaria]. Nauchni Trudove Vissh Selskost. Inst. "Vasil Kolarov" 32 (4): $71-78$ (in Bulgarian). [37]

St oich ev G. T. 1990. [Polyporaceae s.l. in Bulgaria]. Ph.D. thesis (msc). Vissh Selskostopanski Institut Vasil Kolarov, Plovdiv (in Bulgarian). [38]

Stoichev G.T. 1995. [Phellinus Quél. (Hymenochaetaceae Donk) in Bulgaria]. (In:) Jubilee Research Session, Vissh Selskostopanski Institut "Vasil Kolarov", Plovdiv, October, 1995, 4 (1): 221-227. Vissh Selskostopanski Institut "Vasil Kolarov", Plovdiv (in Bulgarian). [39]

Stoichev G. T., Dimcheva D. M. 1987. [New taxa and chorological data for the fungus flora of Bulgaria]. Fitologiya 33: 67-69 (in Bulgarian). [40]

Stoichev G., Dimcheva M. 1988. [New mushrooms for the Pirin mountain and Bulgaria]. Nauchni Trudove Vissh Selskost. Inst. "Vasil Kolarov" 33(4): 89-93 (in Bulgarian). [41]

Stoichev G., Naidenov Ya. 1984. [Ganoderma in Bulgaria]. Gorskost. Nauka 3: 83-87 (in Bulgarian). [42]

Vanev S. G., Reid D. A. 1986. New taxa and chorologic data for the Bulgarian fungus flora. Fitologiya 31: 63-70. [43] 